\title{
Shape and morphology of the particles surface of electroerosive powders of micro- and nanometric fractions, obtained from H17MYuA steel in kerosene
}

\author{
E.V. Ageev ${ }^{1}$, S.V. Khardikov ${ }^{1, *}$, E.A. Vorobyev $^{1}$, A.A. Sysoev ${ }^{1}$ \\ ${ }^{1}$ FGBOU VO Southwest state University, 9450 years of October street, Kursk, 305040, \\ Russia.
}

\begin{abstract}
One of the main directions of engineering technology development at present is the improvement of existing and the development of new waste-free, environmentally friendly, material-saving production processes. Powder metallurgy is a branch of technology, including the manufacture of powders from metals and their alloys and the preparation of blanks and products from them without melting the main component. In most cases, new materials are created in order to provide the optimal combination of product price and operational characteristics. Chromium is the most common alloying element. Steel alloyed with chromium has increased strength, hardness, wear resistance, resistance to corrosion in aggressive and oxidizing environments. The presence of chromium increases the hardenability of steel. The process of obtaining powder materials by the method of electroerosive dispersion compares favorably with the possibility of producing powders even from particularly hard and refractory materials. In the case of grinding material by this method, the only important criterion is the electrical conductivity of the material being ground. Worn parts and other scrap can be used as feedstock during the process. The purpose of the study is to investigate the powder obtained from the waste of chromium-containing steels. Thanks to the study of the used powder samples on modern devices, results were obtained, which indicate that the chromium-containing powder, obtained by electroerosive dispersion, can be reused in the restoration and hardening of machine parts.
\end{abstract}

\section{Introduction}

Currently, progress in the field of product quality and increasing the productivity of technological processes is associated with the use of nanopowders. In the near future, nanotechnology and nanosized materials will determine the fate of technological progress. The rapid development of powder metallurgy and the continuously expanding range of nanomaterials and products from them stimulate the study of their structure and properties.

\footnotetext{
* Corresponding author: hardikov1990@mail.ru
} 
H17MYuA stainless steel is used in all areas of production where corrosion resistance at high temperatures is required, as well as where there is no need to use more expensive nickel-containing stainless steel grades. Therefore, the acute problem of processing its waste into powders suitable for industrial use.

One of the promising methods for producing powders from virtually any conductive material, including H17MYuA steel waste, is characterized by relatively low energy costs, process safety and environmental friendliness, the absence of mechanical wear of the equipment, and the particles production of predominantly spherical shape ranging in size from several nanometers to hundreds micron is a method of electroerosive dispersion (EED) [1-7].

The EED process is the destruction of a conductive material as a result of local exposure to short-term electrical discharges between electrodes and (or) alloy particles (electrical erosion of the material). In the discharge zone, under the influence of high temperatures, heating, melting, and partial evaporation of the material occur. Liquid material and vaporous material are ejected into the working fluid and solidifies in it with the formation of individual particles.

The physico-mechanical properties of the powders, obtained from H17MYuA steel wastes, due to which they can be widely used in the technological processes of manufacturing, restoration, and hardening of machine parts and tools, are determined by their granulometric, X-ray structural, chemical composition, surface shape and morphology, and microhardness of particles.

The aim of this work was to study the shape and morphology of the powder particles surface, obtained by electroerosive dispersion from steel waste X17MUA in lighting kerosene.

\section{Materials and methods}

To carry out the planned studies, H17MYuA steel waste was selected. As a working fluid, kerosene lighting was chosen. To obtain the powder by the method of electroerosive dispersion, we used a device for EED of conductive materials. The waste was loaded into a reactor filled with a working fluid - lighting kerosene, the process was carried out with the following electrical parameters: capacity of discharge capacitors $55 \mu \mathrm{F}$, voltage $120 \ldots 130$ $\mathrm{V}$, pulse repetition rate $95 \ldots 100 \mathrm{~Hz}$. As a result of local exposure to short-term electrical discharges between electrodes, the waste material was destroyed with the formation of dispersed powder particles.

To obtain powder from wastes by the method of electroerosive dispersion, we used a device for EED of conductive materials developed by the authors [8, 9]. As a result of local exposure to short-term electrical discharges between the electrodes, the waste material was destroyed with the formation of dispersed powder particles.

The EED process is the destruction of conductive material as a result of local exposure to short-term electrical discharges between the electrodes. In the discharge zone, under the influence of high temperatures, heating, melting, and partial evaporation of the material occur. To obtain high temperature in a limited area of small volume, a large concentration of energy is required. Achieving this goal is carried out using pulsed voltage, and EED is carried out in a liquid medium (working fluid), which fills the gap between the electrodes, called the inter-electrode gap (IEG).

Due to the fact that any smooth surface has its own macro- or microrelief, there will always be two points between two electrodes, the distance between which will be less than between other points of the electrode surfaces. When a source of electric current is connected to the electrodes, a current begins to flow between the electrodes, and an electric field appears, the intensity of which in the area where the gap between the electrodes is 
minimal will reach its maximum value. The location of this section depends on local protrusions, irregularities on the electrodes, on the presence and size of electrically conductive particles located in the inter-electrode gap. To describe the processes occurring during an electric discharge, we will divide them into three stages.

The first stage of the EED process is the breakdown of the inter-electrode gap (formation of a discharge channel) as a result of the formation of a zone with high field strength. Under the action of the discharge, the working fluid is ionized in the gap through which electric current begins to flow. The second stage is the formation of a gas bubble near the discharge channel from liquid and metal vapor, and heating of the electrodes. The development of a discharge in the inter-electrode gap is accompanied by the appearance of a shock thermal wave, which occurs in the initial stages of the discharge and leads to the formation of a gas bubble in the working fluid. The third stage will be the cessation of current, separation of the shock wave from the gas bubble and the continuation of its expansion by inertia, the evacuation of erosion products.

To study the shape and morphology of the images, photographs were taken on a Quanta 600 FEG scanning electron microscope. Using scanning electron microscopy, it is possible to directly analyze powder particles with a fairly high resolution. In a scanning electron microscope, a large depth of focus is achieved, which allows one to observe a threedimensional image of the studied structure.

The FEI Quanta 600 FEG is a scanning electron microscope (SEM) with a field emission cathode and integrated EDAX TRIDENT XM 4 integrated microanalysis system, consisting of an energy-dispersive X-ray spectrometer (EDS), a wave dispersive spectrometer X-ray radiation (WDS) and systems for analyzing the structure and texture of crystalline materials by the method of reflection electron diffraction (EBSD). The microscope allows you to receive images of various objects with an increase exceeding 100,000 times, with a large number of image elements (pixels). It is designed to carry out various studies with minimal time for preparation of objects, because Quanta 600 FEG allows you to work with a variety of types of samples, including non-conductive, contaminated, wet or capable of gas evolution under vacuum. This ensures exceptional image depth of field.

QUANTA 600 FEG (manufacturer FEI (Holland)) - an electron-ion scanning microscope with an electron beam column, equipped with a tungsten cathode, accelerating voltage from $200 \mathrm{eV}$ to $30 \mathrm{kV}$, resolution (with optimal WD) $3.5 \mathrm{~nm}$ at $35 \mathrm{kV}$; $3.5 \mathrm{~nm}$ at 30 $\mathrm{kV}$ in the natural environment; $<15 \mathrm{~nm}$ at $1 \mathrm{kV}$ in low vacuum mode. Magnum ion column with a gallium liquid-metal ion source, accelerating voltage from $5 \mathrm{kV}$ to $30 \mathrm{kV}$, resolution $20 \mathrm{~nm}$. The system is equipped with a 5-axis motorized table 50x50x25 mm, gas injection systems for spraying conductors and dielectrics, as well as for etching samples.

The microscope allows you to obtain images of various objects with an increase exceeding 100,000 times, with a large number of decomposition elements (pixels). It is designed to carry out various studies with minimal time for preparation of objects, ensuring their observation with an exceptional depth of field.

\section{The study of the powders microstructure}

Using scanning electron microscopy (SEM) with a secondary electron detector, particles of a powder sample were studied (Fig. 1).

It is shown that the shape of the powder particles is due to the form in which the material is ejected from the hole during the EED. It can be seen that particles having a regular spherical or elliptical shape prevail in the powder. They are obtained by crystallization of the molten material (liquid phase). Particles, formed during crystallization of a boiling material (vapor phase), have an irregular shape, an order of magnitude smaller 
than the particles, formed by their liquid phase, and usually agglomerate with each other and on the surface of other particles. In the EED process, such particles are most susceptible to chemical and phase changes.

It has been established that particles, ejected from the well in the solid state (solid phase), are formed under the action of shock waves of the discharge channel and under the influence of thermal stresses, as well as particles of the solid phase are formed upon brittle fracture of sharp edges and edges of the dispersible material when it is mixed during the EED process. Such particles, as a rule, have an irregular fragmentation shape, sometimes with fused faces and edges.

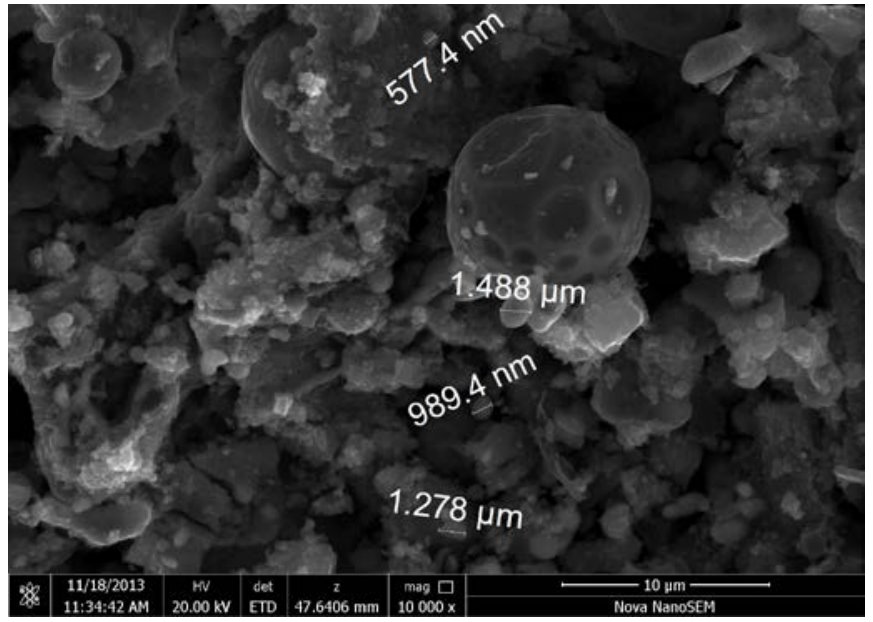

Fig. 1. The image of powder particles, obtained by EED in kerosene (magnification 10000)

\section{Conclusion}

In the course of experimental studies aimed at studying the particle size distribution of a chromium-containing powder, obtained by electroerosive dispersion of waste in lighting kerosene with the following electrical parameters: capacity of discharge capacitors $55 \mu \mathrm{F}$, voltage $120 \ldots 130 \mathrm{~V}$, pulse frequency $95 \ldots 100 \mathrm{~Hz}$; were determined: average particle size, specific surface area, which will determine the rational area of their practical application.

Based on the obtained results, it can be concluded that the powder, obtained by electroerosive dispersion from steel $\mathrm{H} 17 \mathrm{MYuA}$ waste, can be reused in the restoration and hardening of machine parts.

The reported study was funded by RFBR, project number 19-33-90053.

\section{References}

1. A. p. 70000 USSR, B 22f, 09/00 A method for producing powders and a device for its implementation / B.R. Lazarenko, N.I. Lazarenko (USSR). - No. 1371/321510; declared 04/01/1943; publ. 09/23/1964, Bull. 22, 2.

2. Gusev, V.N. Anodic-mechanical processing of metals (1952) 321 p.

3. Janitor M.I. Development of physico-chemical and technological fundamentals of the processing of tungsten-cobalt hard alloy by electroerosive dispersion [Text]: diss. ... cand. tech. Sciences / Dvornik Maxim Ivanovich. - Khabarovsk, (2006) . - 116 p. 
4. E.V. Ageev, Hardening technologies and coatings, (2011), 6, 8-14.

5. R.A. Latypov, G.R. Latypova, E.V. Ageev, P.I. Burak, International Scientific Journal, (2013), 5, 80-86.

6. E.V. Ageev, Technology of metals. - (2012), 9, 36-45.

7. Ageev E.V. Obtaining powders from solid alloy waste by the method of electroerosive dispersion / E.V. Ageev // Electrometallurgy. - (2011), 10, 24-27.

8. E.V. Ageev Patent 2449859, Russian Federation, C2, B22F9 / 14. Installation for producing nanodispersed powders from conductive materials, applicant and patent holder Southwestern State University. - No. 2010104316/02; application 02/08/2010; publ. 05/10/2012. - 4 p.

9. E.V. Ageev, E.V. Ageeva // Bulletin of mechanical engineering. - (2013) 11. 51-57. 